\title{
ROMAN LAW AND PRINCIPLES OF THE CONSTITUTION OF THE ROMAN REPUBLIC OF 1849 IN THE SCIENTIFIC AND EUROPEAN CONSTITUTIONAL CONTEXT. THE ROOTS OF THE CRISIS OF CONSTITUTIONALISM
}

\author{
DIRITTO ROMANO E PRINCIPI DELLA COSTITUZIONE \\ DELla REPUBBLICA ROMANA DEL 1849 NEL \\ CONTESTO SCIENTIFICO E COSTITUZIONALE \\ EUROPEO. Alle RADICI DELla CRISI DEL \\ COSTITUZIONALISMO
}

\begin{abstract}
Given that contemporary European constitutionalism has its roots essentially in the "Déclarations" developed during the French Revolution and, to some extent, also in the 19th century experience of the codifications, and that the codes, in turn, are inspired by Roman law, it is worth asking how much of Roman law there is in the constitutions and statutes approved during the $19^{\text {th }}$ century. Therefore, turning the question particularly to the subject of the Constitution of the Roman Republic of 1849, the work of the constituents demonstrates the existence of a sort of laboratory, political and juridical, within which a synthesis between the Roman juridical tradition and the tripartite separation of powers was sought.
\end{abstract}

\section{SOMMARIO}

Posto che il costituzionalismo europeo contemporaneo affonda le sue radici essenzialmente nelle "Déclarations" elaborate nel corso della rivoluzione francese e, per certi versi, anche nellesperienza ottocentesca delle codificazioni e che i codici, a loro volta, si ispirano al diritto romano, cè da chiedersi quanto del diritto romano vi sia nelle costituzioni e negli statuti approvati nel corso del XIX secolo. Quindi, rivolgendo particolarmente la questione al tema della Costituzione della Repubblica Romana del 
1849, i lavori dei costituenti dimostrano lesistenza di una sorta di laboratorio, politico e giuridico, nellambito del quale si è cercata una sintesi tra la tradizione giuridica romana e la separazione tripartita dei poteri.

KEYWORDS: constitution, Roman republic, Roman law, constitutionalism.

Parole Chiave: costituzione, repubblica romana, diritto romano, costituzionalismo.

\section{LA Costituzione Della RePubblica Romana DEL 1849. IL CONTESTO SCIENTIFICO E COSTITUZIONALE EUROPEO}

Posto che il costituzionalismo europeo contemporaneo affonda le sue radici essenzialmente nelle "Déclarations" elaborate nel corso della rivoluzione francese e, per certi versi, anche nell'esperienza ottocentesca delle codificazioni e che i codici, a loro volta, si ispirano al diritto romano, cê da chiedersi quanto del diritto romano vi sia nelle costituzioni e negli statuti approvati nel corso del XIX secolo. Quindi, rivolgendo particolarmente la questione al tema della Costituzione della Repubblica Romana del 1849, viene da chiedersi: i costituenti, che certamente conoscevano la storia romana, quanto conoscevano del diritto pubblico romano?

Innanzitutto, un dato certo. Al tempo, nell'Università romana de 'La Sapienza' non era insegnata la storia del diritto romano. Può apparire inverosimile, considerata anche la localizzazione dell'Ateneo, ma è così; l'insegnamento di questa disciplina, infatti, solo nel 1847 era stato oggetto di rivendicazione da parte degli studenti romani (Spano, N., 1935) ${ }^{1}$.

Poi, una considerazione. Il complesso contesto europeo di quegli anni fu influenzato da determinate opere che, più di altre, seppero dirigere le riflessioni degli intellettuali. Particolare rilievo per gli studi giuridici ebbe la monumentale opera romanistica di un grande studioso tedesco che proprio ai movimenti del 1848 aveva preso parte, patendone poi le conseguenze: Theodor Mommsen (Fraccaro, P., 1934, pp. 594-595). Tuttavia, la lettura in chiave giuridica della storia romana fu tracciata da Mommsen nel 1854-1856, nella prima edizione della sua storia di Roma, iniziata nel 1850 (Mommsen. Th., 1854-1856) ${ }^{2}$. Circa quindici anni dopo, apparve la prima edizione 
dellopera dedicata specificamente al diritto pubblico romano (Mommsen, Th., 1871). Si trattava certamente di opere destinate a influenzare gli studi romanistici, anche a causa della impareggiabile scienza del grande Maestro; esse però furono pubblicate successivamente al periodo 1848-1849.

Questo significa che nel 1849 la conoscenza del diritto pubblico romano passava attraverso altri autori, altre opere (Koschaker, P., 1947); Orestano, R., 1987) ${ }^{4}$. Particolare rilievo, per gli studi pubblicistici del tempo, avevano le riflessioni di Niccolò Machiavelli (Machiavelli, N., 1531) e di Giambattista Vico (Vico, G., 1744) ${ }^{6}$, la visione critica verso il diritto romano da parte di Montesquieu (Montesquieu, 1748) ${ }^{7}$ e la risuscitazione della romanità da parte di Jean-Jacques Rousseau (Rousseau, J.-J., 1762) ${ }^{8}$, nonché il superamento delle idealità degli antichi ad opera di Henri-Benjamin Constant (Constant, H. B., 1819) fino alle indagini storiche condotte da Barthold Georg Niebuhr (Niebuhr, B. G., 1811; Momigliano, A., 1934) $)^{9}$ e alle elaborazioni teorico-pratiche prodotte da Friedrich Karl von Savigny (von Savigny, F. K., 1815-1831, 1840-1849) e dalla pandettistica. Comunque, va detto che i testi composti in lingua francese erano più facilmente letti e quindi maggiormente conosciuti rispetto alle opere di autori tedeschi.

A questo punto, piuttosto che porre la questione se i costituenti romani del 1849 conoscessero gli istituti del diritto pubblico romano, occorre interrogarsi circa la 'visione' sistematica tramite la quale ne avevano preso coscienza.

\section{PRINCIPI DELLA BOZZA E DEL TESTO DELLA COSTITUZIONE ROMANA DEL 1849}

Per provare a dare una risposta, è necessario analizzare alcuni principi presenti nella bozza di Costituzione della Repubblica Romana del 17 aprile 1849 (che chiamerò 'Bozza') e nel testo della Costituzione approvata il 1 luglio e promulgata il 3 luglio 1849 (che chiamerò 'Testo'). Trattandosi di costituzione mai applicata, entrambe le redazioni sono utili per comprendere il movimento costituzionalista del tempo, nonché il rapporto tra esso e il diritto pubblico romano (in maggior misura repubblicano).

Mi occuperò, specificamente, dei principi emergenti all'interno di alcuni grandi temi, incentrati sul concetto di repubblica relazionato ad altri concetti, quali divinità, poteri e responsabilità. Per ogni tema, procedendo da una 
sorta di 'massima' enucleata dalle fonti romane, porrò a confronto articoli della Bozza con articoli del Testo.

\section{Nozione di repubblica}

La 'massima' romana di riferimento, circa la nozione di repubblica, è tratta dallopera di Cicerone dedicata, appunto, alla repubblica.

- Cic. Rep. 1.25.39: est igitur res publica res populi [quindi, la repubblica è cosa del popolo].

Tramite il brano di Cicerone, è definito lo strettissimo e imprescindibile rapporto tra popolo e repubblica.

Dalla Bozza possiamo estrarre i principi contenuti in:

- art. 1 dei 'Principj fondamentali', per il quale il popolo si è "costituito" in Repubblica;

- art. 6 dei 'Principj fondamentali', per cui la Repubblica è difesa militarmente da tutti i suoi cittadini;

- artt. 16, 63 e 67, per cui il popolo elegge direttamente: l'Assemblea, che ha il potere legislativo, ma partecipa anche a quello esecutivo e giudiziario attraverso la scelta dei membri del Consiglio di Stato (art. 63), che a loro volta propongono ai Consoli i giudici da nominare (art. 67); i Consoli, che possiedono il potere esecutivo, ma partecipano anche al potere giudiziario attraverso la nomina dei giudici (art. 67); i Tribuni (art. 16).

Dal Testo possiamo estrarre i principi contenuti in:

- art. I, per il quale il popolo è "costituito" in Repubblica;

- art. 15, per cui ogni potere "viene" dal popolo;

- art. 20, per cui il popolo elegge l’Assemblea.

La centralità del popolo è evidente in entrambi i testi. Tuttavia, il disegno tracciato dalla Bozza presenta una maggiore effettività quanto all'esercizio di poteri; nel Testo, invece, il minor grado di effettività del potere popolare pare bilanciato dall'espresso richiamo alla provenienza popolare dei poteri, di tutti i poteri. 


\section{Repubblica e divinità}

Le 'massime' romane di riferimento sono tratte dallopera di Cicerone dedicata alle leggi e da una costituzione degli imperatori Graziano, Teodosio I e Valentiniano II del 380 (cd. editto di Tessalonica).

- Cic. Leg. 1.23: universus hic mundus una civitas communis deorum atque hominum [questo mondo intero è una comune città degli dèi e degli uomini];

- C.Th. 16.1.2: cunctos populos, quos clementiae nostrae regit temperamentum, in tali volumus religione versari, quam divinum Petrum apostolum tradidisse Romanis [vogliamo che tutti i popoli, da noi governati secondo moderazione e umanità, professino il culto di quella religione, che l'apostolo Pietro tramandò ai Romani].

Le due 'massime' definiscono il rapporto tra umano e divino, l'una nell'età repubblicana, l'altra più di sessant'anni dopo il cosiddetto editto di Milano dell'anno 313. Nella prima è posta in rilievo una sorta di comunanza di ambiti tra umano e divino, entrambe parti di un sistema (giuridico-religioso) unitario entro una professione politeista, in assenza di mediazioni da parte di entità terze, come esemplarmente lo stato. Nella seconda, l'unità del sistema è garantita dall'unica professione monoteista, ove il rapporto tra popolo e Divinità è mediato dalle figure del principe degli apostoli e dell'imperatore.

Dalla Bozza possiamo estrarre i principi contenuti in:

- art. 8 dei 'Principj fondamentali', per cui vè una religione (monoteista) dello Stato, che deve essere inteso quale entità di mediazione tra popolo e Divinità;

- art. 32, per cui, in relazione alla promulgazione delle leggi, è tracciato direttamente il rapporto tra popolo e Divinità, senza la mediazione di organismi come lo stato;

- art. 69, per cui lo stesso rapporto diretto tra popolo e Divinità è tracciato con riferimento all'amministrazione della giustizia.

Dal Testo possiamo estrarre i principi contenuti in: 
- art. 32, per cui, in relazione alla promulgazione delle leggi, è tracciato direttamente il rapporto tra popolo e Divinità, senza la mediazione di organismi come lo stato.

Nella Bozza, è evidente il tentativo di conciliare il pluralismo repubblicano (che per i romani era politeista) con il centralismo imperiale monoteista. Tentativo, peraltro, abbandonato nel Testo, ove domina il pluralismo repubblicano (seppur ormai monoteista).

\section{Repubblica e poteri}

Le 'massime' romane di riferimento sono tratte dall'orazione di Cicerone sulla proposta di legge agraria del tribuno Servilio Rullo del 63 a.C. e dal primo libro delle Istituzioni del giurista Ulpiano dei primi decenni del III secolo (come raccolta nei Digesti di Giustiniano).

- Cic. Leg. agr. 2.7.17: omnes potestates, imperia, curationes ab universo populo Romano proficisci convenit [tutti i poteri, gli imperi, le cure si conviene che provengano dall'intero popolo romano];

- Ulp. in D. 1.4.1 pr.: populus ei et in eum (principi et in principem sc.) omne suum imperium et potestatem conceferat [il popolo conferisce a lui e in lui (sc. al principe e nel principe) ogni proprio impero e potestà].

Entrambe le 'massime' evidenziano il fondamento popolare dei poteri, sia repubblicani sia imperiali, laddove è sempre il popolo ad attribuirli, anche all'imperatore.

Dalla Bozza possiamo estrarre i principi contenuti in:

- art. 1 dei 'Principj fondamentali', per cui il fondamento dei poteri è nel popolo, in quanto sovrano per diritto eterno;

- $\operatorname{artt} .9,11,16,32,47,69$ e 70, per cui l'esercizio dei poteri può essere del popolo, sia direttamente come tutto (artt. 11, 16, 26) o come parte (artt. 9, 47 e 70), sia in forma mediata, cioè in suo nome (artt. 32 e 69) ovvero attraverso altri organismi previamente eletti dal popolo (artt. 37 e 66).

Dal Testo possiamo estrarre i principi contenuti in:

- artt. I e 15, per cui il fondamento dei poteri è nel popolo, in quanto questi è sovrano per diritto eterno e da questo viene ogni potere; 
- artt. 10, 12, 20, 32, 49, 52 e 53, per cui l'esercizio dei poteri può essere del popolo, sia direttamente come tutto (artt. 12 e 20) o come parte (artt. 10 e 53), sia in forma mediata, cioè in suo nome (artt. 32 e 52) ovvero attraverso altri organismi previamente eletti dal popolo (art. 49).

L'esercizio diretto del potere da parte del popolo appare maggiormente pronunciato nella Bozza rispetto al Testo. Quasi come una conseguenza di tale pronunciamento, lo schema della separazione dei poteri è tracciato meno nitidamente nella Bozza, rispetto al Testo. Senza dubbio, la previsione del Tribunato nella Bozza, ma non nel Testo, condiziona il disegno costituzionale quanto alla separazione dei poteri. Infatti, il Tribunato più del popolo pare costituire un elemento in grado di compromettere l'equilibrio nell'assetto dei poteri, nella logica della loro separazione.

\section{Repubblica e responsabilità (dell'esecutivo)}

Le 'massime' romane di riferimento sono tratte dallopera sulle leggi di Cicerone e dal quarantaduesimo libro sull'editto pretorio del giurista Ulpiano (come raccolta nei Digesti di Giustiniano).

- Cic. Leg. 3.16: ut ei (consuli sc.) reliqui magistratus omnes pareant, excepto tribuno [tutti gli altri magistrati devono obbedire al console, eccetto il tribuno];

- Ulp. in D. 47.10.32: nec magistratibus licet aliquid iniuriose facere. Si quid igitur per iniuriam fecerit magistratus vel quasi privatus vel fiducia magistratus, ... potest conveniri... Si is magistratus est, qui sine fraude in ius vocari non potest, exspectandum esse, quoad magistratu abeat [neppure ai magistrati è lecito fare qualcosa contrariamente al diritto. Se dunque il magistrato farà qualcosa di contrario al diritto, o come privato o in qualità di magistrato, può essere citato in giudizio... Se si tratta di un magistrato che non può essere convenuto in giudizio senza danno, sia piuttosto da attendere che esca dalla carica].

Dalle 'massime' si evince un rapporto gerarchico tra consoli e altri magistrati, nonché la responsabilità dei magistrati per le azioni compiute come tali e come privati, rilevabile da parte di chi ha subito il danno. Circa 
i tempi dellazione, è da notare la prevalenza dell'interesse pubblico al buon andamento dellattività amministrativa e di governo, tale da impedire l'esercizio tempestivo dell'azione contro il magistrato; il che denota una certa discrezionalità nell'avviamento del giudizio.

Dalla Bozza possiamo estrarre i principi contenuti in:

- art. 56, per cui l'accusa contro i Consoli, teminato il loro mandato, è iniziativa dei Tribuni;

- art. 47, per cuilaccusa contro iConsoli, durante il loro mandato, èiniziativa dei rappresentanti del popolo ovvero di un qualsivoglia cittadino;

- art. 48, per cui la sospensione dei Consoli accusati dipende dalla discrezionalità dell'Assemblea;

- art. 72, per cui il giudizio si svolge innanzi a un tribunale censorio composto da togati.

Dal Testo possiamo estrarre i principi contenuti in:

- art. 44, per cui l'accusa contro i Consoli, durante il loro mandato, è iniziativa dei rappresentanti del popolo;

- art. 45, per cui la sospensione dei Consoli accusati è obbligatoria;

- art. 55, per cui il giudizio si svolge innanzi a un tribunale supremo, composto da togati e da cittadini.

Nella Bozza, oltre al potere dei Tribuni, è da evidenziare il maggiore spazio attribuito al potere popolare, diffuso pur se condizionato (art. 47), ovvero mediato e concentrato in specifici organismi eletti dal popolo, quali il Tribunato e l'Assemblea, laddove emerge la discrezionalità (dell'Assemblea) circa la sospensione dalla carica del Console accusato.

Nel Testo, oltre allassenza del Tribunato, occorre rilevare che il potere popolare viene spostato dal momento dell'iniziativa accusatoria a quello del giudizio, laddove infatti una parte dei giudici è tratta da apposite liste popolari. Da notare, altresì, lobbligatorietà della sospensione del Console accusato. 


\section{Conclusioni}

Alla luce di queste brevi riflessioni, concentrate su alcuni principi rilevabili nella Bozza e nel Testo, mi sembra evidente una certa attenzione ai temi del diritto pubblico romano o, per semplificare, alla storia del diritto romano. Al di là della suggestione che il nome di alcune cariche suscita, come ad esempio Consolato e Tribunato, si pone alla nostra attenzione il contenuto di talune disposizioni. Da esse, infatti, emergono caratteri riconducibili ad alcuni principi del diritto romano, come sintetizzati nelle 'massime' che ho selezionato e riportato nel testo. È altresì evidente, però, una forte tensione tra i costituenti circa l'adesione a istituti repubblicani o (anche) imperiali romani. La prova più evidente di tale tensione è nell'istituto del Tribunato, presente nella Bozza ed espunto nel Testo; è, questo, il caso più eclatante, ma non l'unico. Si confrontino, infatti, le 'massime' romane con quanto disposto nella Bozza e nel Testo; emergono differenze, talvolta anche profonde. E tali differenze dipendono soprattutto dal principio posto a fondamento sia della Bozza sia del Testo: la separazione dei poteri.

Orbene, tornando alla questione dalla quale mi sono mosso, cosa dire circa la 'visione' sistematica tramite la quale i costituenti del 1849 avevano preso coscienza del diritto pubblico romano?

Certo è che il tentativo di conciliare il pensiero di Montesquieu con quello di Rousseau non aveva prodotto effetti significativi. La cancellazione del Tribunato nel Testo ne costituisce una delle prove decisive.

$\mathrm{Al}$ contempo, però, pareva superato un certo ipercriticismo verso il diritto (pubblico) romano. Evidentemente, i lavori dei costituenti del 1849, pur tra mille difficoltà e sotto l'assedio di quattro corpi di spedizione, dimostravano l'esistenza di una sorta di laboratorio, politico e giuridico, nell'ambito del quale ci si sforzava di trovare una sintesi tra la tradizione giuridica romana e la separazione tripartita dei poteri. Era tempo che gli intellettuali europei intervenissero, prendendo una posizione chiara soprattutto verso la millenaria esperienza giuridica romana.

L'entrata in campo di Mommsen nel 1850, con l'inizio di una pluridecennale, imponente e celebratissima rivisitazione della storia di Roma, esaminata sotto vari profili e pubblicata in vari volumi, varie edizioni e varie lingue ${ }^{10}$, dimostra quanto fosse importante per l'anima liberale della cd. "primavera 
dei popoli" (ri)disegnare il diritto pubblico romano, sulla base del concetto di 'Staat' (Mommsen, Th., 1887, p. 3) ${ }^{11}$. Cề da chiedersi insomma, e ora è giunto il momento di farlo davvero, quanto abbiano influito le vicende della Repubblica Romana del 1849 sul pensiero del premio Nobel Theodor Mommsen (Buonocore, M. (a cura di); Baruffa, A., 1994, pp. 197-200 e p. 212$)^{12}$.

\section{Articoli della Bozza citati nei paragrafi precedenti}

1. La sovranità essendo per diritto eterno nel Popolo, il Popolo dello Stato Romano si è costituito in Repubblica.

6. Tutti i cittadini debbano difendere fin colla vita la Repubblica e la indipendenza nazionale.

8. La Religione Cattolica è la Religione dello Stato.

9. Il diritto di petizione è di ciscuno e di tutti.

11. Tutti i cittadini compiuti gli anni 18 appartengono alla guardia nazionale nei modi e colle eccezioni stabilite da una legge.

16. Il popolo elegge i suoi Rappresentanti, i consoli, ed i Tribuni in Comizi generali.

32. Le leggi sono proposte da cinque Rappresentanti, o dal Consolato; adottate dall'Assemblea sono promulgate dal Presidente di essa in nome di Dio e del Popolo, e rimesse al Consolato per l'esecuzione.

47. I Consoli possono essere posti in stato d'accusa dall'Assemblea sulla dimanda di cinque Rappresentanti, o su petizione di chiunque del popolo. La dimanda deve essere discussa come una legge per tre volte senza intervento del Tribunato all'intervallo almeno di un giorno fra una discussione e l'altra.

48. Se viene ammesso lo Stato di accusa, l'Assemblea deve insieme decidere se i Consoli debbano restar sospesi dalle loro funzioni. Se decide per l'affermativa, l'ufficio Consolare è trasferito a tre Tribuni da scegliersi immediatamente dal tribunato stesso.

56. I Consoli, cessata la loro gestione, rendono conto al Tribunato. Ai Tribuni appartiene o l'approvazione o la proposta di accusa.

63. (il Consiglio di Stato) Ė composto di quindici Consiglieri tratti dalle varie province, e scelti dall'Assemblea sopra terne proposte dal Tribunato. 
67. (i Giudici) Nominati dai Consoli sulla proposta del Consiglio di Stato sono inamovibili, né possono essere promossi che sulla proposta dello stesso Consiglio, né sospesi degradati o destituiti se non dopo regolare procedura e Sentenza.

69. La giustizia è amministrata in nome di Dio e del Popolo pubblicamente...

70. Nelle cause criminali al popolo appartiene il giudizio del fatto, ai Tribunali l'applicazione della legge. La istituzione dei Giudici del fatto è determinata dalla legge relativa.

72. È pure istituito un tribunale censorio per decidere dei delitti del potere esecutivo in ciò che riguarda l'officio di esso. Il Tribunale censori si compone di sette Giudici, dei quali quattro sono desunti dal Tribunale di Cassaizione e tre dal Tribunale d’appello di Roma, tutti per turno a trimestre.

\section{Articoli del Testo citati nei paragrafi precedenti}

1. La sovranità è per diritto eterno nel Popolo. Il Popolo dello Stato Romano è costituito in Repubblica democratica.

10. Il diritto di petizione può esercitarsi individualmente e collettivamente.

12. Tutti i cittadini appartengono alla Guardia Nazionale nei modi e colle eccezioni fissate dalla legge.

15. Ogni potere viene dal Popolo. Si esercita dall'Assemblea, dal Consolato, dall'Ordine giudiziario.

20. I comizi generali si radunano ogni tre anni nel 21 aprile. Il Popolo vi elegge i suoi Rappresentanti con voto universale, diretto e pubblico.

32. Le leggi adottate dall'Assemblea vengono senza ritardo promulgate dal Consolato in nome di Dio e del Popolo. Se il Consolato indugia, il presidente dell'Assemblea fa la promulgazione.

44. I Consoli e i Ministri possono essere posti in stato d'accusa dall'Assemblea sulla proposta di dieci Rappresentanti. La dimanda deve essere discussa come una legge.

45. Ammessa l'accusa, il Console è sospeso dalle sue funzioni. Se assoluto, ritorna all'esercizio della sua carica, se condannato, l'Assemblea passa a nuova elezione.

49. I Giudici nell'esercizio delle loro funzioni non dipendono da altro potere dello Stato. 
52. La giustizia è amministrata in nome del Popolo pubblicamente; ma il Tribunale, a causa di moralità, può ordinare che la discussione sia fatta a porte chiuse.

53. Nelle cause criminali al Popolo appartiene il giudizio del fatto, ai Tribunali l'applicazione della legge. La istituzione dei Giudici del Fatto è determinata da legge relativa.

55. Un Tribunale supremo di giustizia giudica, senza che siavi luogo a gravame, i Consoli ed i Ministri messi in istato di accusa. Il Tribunale supremo si compone del Presidente, di quattro Giudici più anziani della Cassazione e di Giudici del Fatto, tratti a sorte dalle liste annuali, tre per ciascuna provincia.

\section{Riferimenti bibliografici:}

Baruffa, A. (1994). Giovanni Battista de Rossi. Larcheologo esploratore delle catacombe, Città del Vaticano, pp. 197-200 e p. 212. ISBN 9788820920104.

Buonocore, M. (a cura di). Le lettere di Theodor Mommsen agli italiani, www. mommsenlettere.org.

Constant, H. B. (1819). De la liberté des Anciens comparée à celle des Modernes.

Fraccaro, P. (1934). s.v. Mommsen, in Enciclopedia Italiana, XXIII, Roma, pp. 594-595.

Koschaker, P. (1947). Europa und das römische Recht, Munchen-Berlin: C.H. Beck (trad. A. Biscardi, L'Europa e il diritto romano, Firenze 1962).

Machiavelli, N. (1531). Discorsi sopra la prima Deca di Tito Livio, Firenze.

Momigliano, A. (1934). s.v. Niebuhr, in Enciclopedia Italiana, XXIV, Roma, p. 799.

Mommsen, Th. (1854-1856). Römische Geschichte, I-III, Leipzig: S. Hirzel.

Mommsen, Th. (1871). Römisches Staatsrecht, I, Leipzig: S. Hirzel.

Mommsen, Th. (1887). Römisches Staatsrecht, III-1, Leipzig: S. Herze, p. 3.

Montesquieu, (1748). De lesprit des loix, Ginevra.

Niebuhr, B. G. (1811). Römische Geschichte, Berlin: Realschulbuchhandlung.

Orestano, R. (1987). Introduzione allo studio del diritto romano, Bologna: Il Mulino. ISBN 9788815009913.

Rousseau, J. J. (1762). Du contract social, Amsterdam.

Spano, N. (1935). L'Università di Roma, Roma 1935, capitolo V. 
Vico, G. (1744). Principi di scienza nuova d'intorno alla comune natura delle nazioni, Napoli.

von Savigny, F. K. (1815-1831). Geschichte des römischen Rechts im Mittelalter, I-VI, Heidelberg: Mohr und Zimmer (II ediz., 1834-1851).

von Savigny, F. K. (1840-1849). System des heutigen römischen Rechts, Berlin: Veit.

\section{Endnotes}

1) I giovani universitari de 'La Sapienza' nel 1847 chiesero l'introduzione della storia del diritto romano nel programma formativo della Facoltà giuridica, perseguendo così la conoscenza delle categorie concettuali romane del diritto pubblico. Si veda, a tal proposito, il capitolo V ("Pio IX e le nuove speranze d'Italia") di Spano, N. (1935).

2) Alla morte di Mommsen, lopera era giunta alla nona edizione ed era stata tradotta nelle maggiori lingue europee. A Milano nel $1863 \mathrm{fu}$ pubblicata la prima traduzione in italiano di G. Sandrini col titolo Storia di Roma.

3) Mommsen fu insignito del premio Nobel per la letteratura nel 1902. Nella motivazione fu espressamente citata l'opera dedicata alla storia di Roma (Römische Geschichte): «the greatest living master of the art of historical writing, with special reference to his monumental work, A history of Rome».

4) Mi limito a indicare due opere, fondamentali per comprendere lo studio del diritto romano fino ai giorni nostri, particolarmente in Europa, nonché gli autori e gli studiosi più influenti anche nellepoca esaminata in questo mio breve contributo.

5) Mi riferisco, in particolare, ai Discorsi sopra la prima Deca di Tito Livio, opera pubblicata postuma nel 1531 .

6) La citata opera di Giambattista Vico fu pubblicata in varie edizioni, di cui l'ultima postuma nel 1744 .

7) Ovviamente, mi riferisco a De l'esprit des loix, testo pubblicato per la prima volta a Ginevra nel 1748.

8) L’opera più rappresentativa è senz'altro Du contract social, pubblicato ad Amsterdam nel 1762.

9) Niebuhr è considerato generalmente quale punto di partenza della metodologia e delle tematiche proprie della dottrina romanistica contemporanea. Niebuhr è altresì noto per aver scoperto nel 1816 il palinsesto di Gaio nella Biblioteca Capitolare di Verona (pur avendolo ritenuto, almeno in un primo momento, attribuibile a Ulpiano). 
10) Lopera di Mommsen sul diritto pubblico romano fu tradotta in francese, a cura di F. Girard e con il titolo Le droit public romain, all'indomani della stampa della III edizione tedesca. La pubblicazione della III edizione fu completata nel 1888 e la pubblicazione della traduzione francese iniziò nel 1889 .

11) Mommsen affermò espressamente che "populus ist der Staat», secondo la sostanziale impostazione di von Savigny.

12) È nota la convinta intensità con cui Mommsen aveva aderito al pensiero liberale (assumendo peraltro una posizione critica verso la Chiesa di Roma). Se ne possono verificare i caratteri di fondo, semplicemente prendendo atto dei contenuti dello scambio epistolare tra Mommsen e il suo intimo amico Giovanni Battista de Rossi, con particolare riferimento ad alcune lettere scambiate nel corso del 1881, a proposito di iniziative politiche volte all'esaurimento del cosiddetto Kulturkampf: «La mia fede politica non è meno santa che la fede religiosa, e questè la disgrazia che il partito cattolico è la rovina della nostra costituzione, e come pare del nostro avvenire liberale. ... la conseguenza sarà, che l'avvenire del partito liberale s'innesta colla rovina del governo papale nella Germania. Avvenire funesto, che noi non vedremo, ma che rimarrà ai nostri poveri bambini» (23 maggio 1881, da Buonocore, M. (a cura di). De Rossi era tra i più intimi amici romani di Mommsen, oltre che tra i più stimati studiosi, fin dagli anni Quaranta del XIX secolo; lo studioso 'danese' (come confidenzialmente definiva se stesso nelle lettere indirizzate all'amico romano), nei suoi numerosi viaggi a Roma era solito frequentare con assiduità la di lui famiglia, entro la quale, peraltro, si manifestava totale dissenso verso l'esperienza repubblicana del 1849 (v. Baruffa, A. (1994). 